\title{
Coastal Monitoring Program of Bathymetry Using Video Images Technique
}

\author{
Muhammad Zikra ${ }^{1, *}$, Noriaki Hashimoto ${ }^{2}$ \\ ${ }^{1}$ Department of Ocean Engineering, Institut Teknologi Sepuluh Nopember, Surabaya, Indonesia \\ ${ }^{2}$ Faculty of Engineering, Kyushu University, Fukuoka, Japan
}

\begin{abstract}
The objective of this research is to investigate the capabilities of optical remote sensing to monitor bathymetry in nearshore area using video images. The technique is designed to extract wave number components based on variation of intensity of brightness at each pixel in the images using cross-spectral correlation approach. This approach is based on pixel array analysis that utilizes a nonlinear inverse method 'Levenberg-Marquardt'. The technique is applied to the data collected at Hasaki beach in Japan from August to December 2006. The results indicate that the estimate of nearshore bathymetry is proved reasonable accurate near shoreline and breaking area where the differences between estimated and survey water depth are less than $10-30 \mathrm{~cm}$.
\end{abstract}

\section{Introduction}

Bathymetry information is very important for coastal and marine engineers to understand the coastal process in nearshore area. Some nearshore activities such as recreation, fishing, navigation, beach nourishment and dredging require the knowledge of bathymetry. Good quality bathymetry information is hence required in order to make clear the physical processes that are taking place. However, combination of traditional in situ survey methods and advanced techniques such as global positioning system and modern ship vehicles are time consuming, and high cost, especially in shallow coastal water area.

The various remote sensing techniques have been applied to derive bathymetry estimate from images. These include measurement of water depth from aerial photograph [1], synthetic aperture radar (SAR) [2], X band radar [3] and SPOT (satellite) images [4] where the sequence of these images can provide a way to record information about changes in seabed topography. Recently, the invention of new digital technology of images form video camera system now can provide and improve an additional capability of automated data collection [5]. This automated data collections have much greater range of time and spatial scales. Also, this technology is suitable for measuring hazardous coastal areas such as surf zone area, where the operations of ship vehicle have limitation on maneuvers.

In these video image data it is possible to see the interaction of the incident wave field with the bathymetry (i.e. wave shoaling and refraction); hence this information can be used to obtain estimates of bathymetry [6][7]. The approach for estimating bathymetry is based on

"Corresponding author: mzikro@oe.its.ac.id 
wave kinematics utilize the depth dependence of the wave speed (or, equivalently, the wavelength and frequency via $\mathrm{c}=\mathrm{f} / \mathrm{k}$, where $\mathrm{c}$ is the wave phase speed, $\mathrm{f}$ is the wave frequency and $\mathrm{k}$ is the wave number $=1 / \mathrm{L}$, and $\mathrm{L}$ is the wavelength). Overall, this approach requires image sequence (or time series of brightness intensity at discretely sampled locations).

Thus, the objective of this research is to investigate the capabilities of optical remote sensing to monitor bathymetry in the nearshore area using video images. The technique is designed to extract wave number components based on variation of brightness intensity at each pixel in the images using cross-spectral correlation approach. This approach based on the pixel array analysis utilizes a nonlinear inverse method 'Levenberg-Marquardt' to find the optimal wavenumber estimate from a model with the observation data set. In this research, the video images measured at Hasaki beach in Ibaragi prefecture in Japan is used to investigate the applicability of the model. In the following sections, we will first review data conditions measured at Hasaki beach area. Next, we summarize mathematical formation of wave number model and inversion method. Finally, we apply the methods to the data, and then draw some conclusions.

\section{Methodology}

In this research, observation was carried out with video camera at Hasaki beach, Japan. The Hasaki beach is located on $120 \mathrm{~km}$ east of Tokyo facing the North Pacific Ocean as shown in Figure 1. In general, Hasaki beach is known as straight sandy coast stretching from north to south with length around $17 \mathrm{~km}$ long. Since 1986, many coastal studies have been conducted in this location especially around the pier which is known as HORS (Hasaki Oceanographical Research Station).

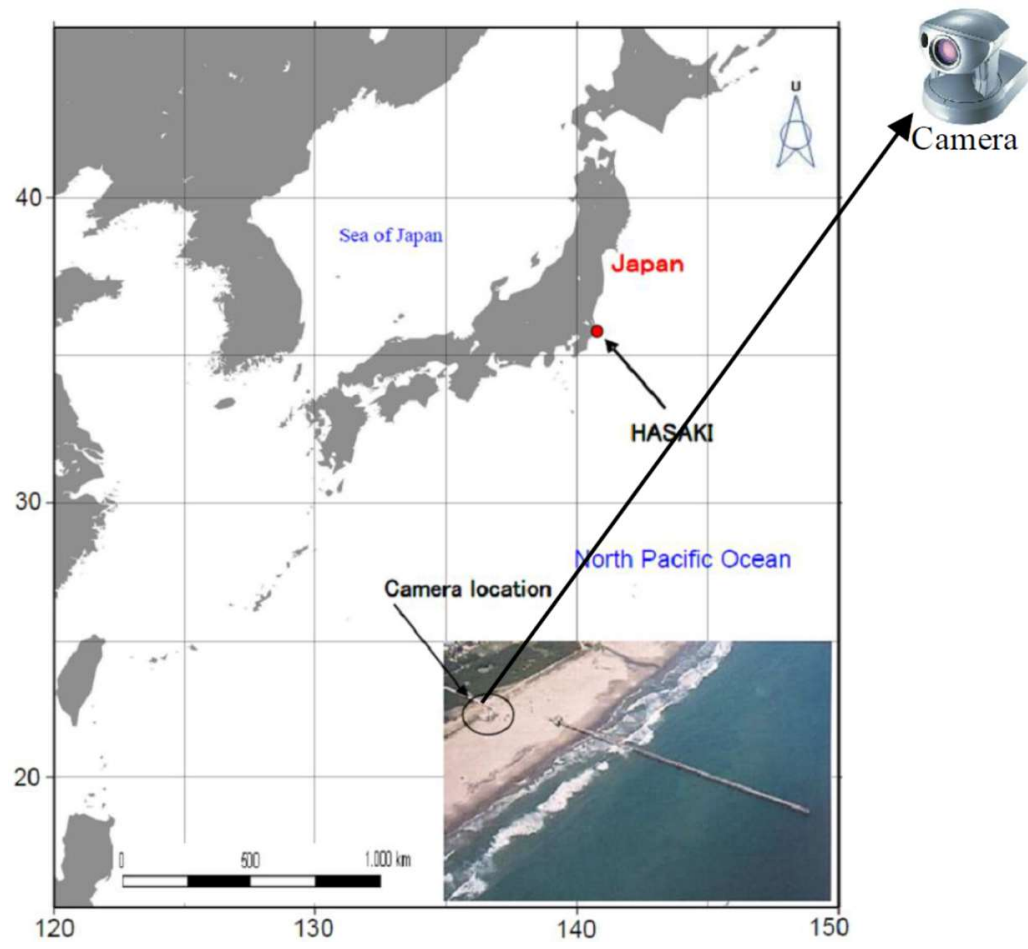

Fig. 1 Study Location 


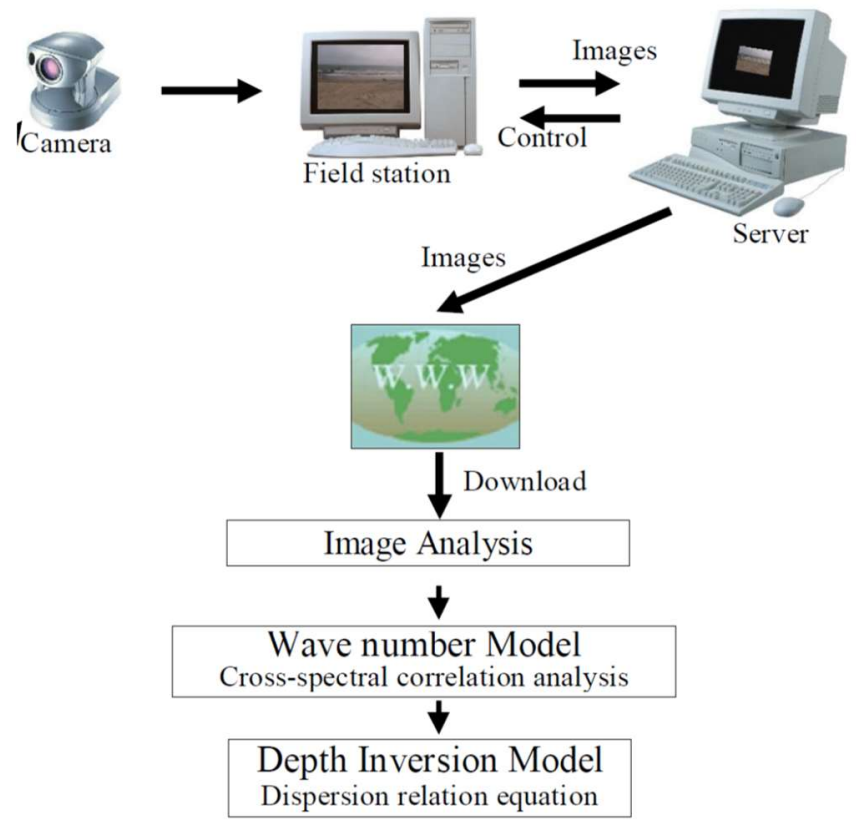

Fig. 2 Methodology of bathymetry monitoring using video images

\subsection{Video Camera System}

The video camera system in Hasaki site was first installed on August 16, 2006. The data sets of video images were collected from single camera network Canon VB-C50iR on $10 \mathrm{~m}$ height above the ground level to generate images with the resolution of $640 \times 480$ pixels. In this video camera system, snapshot images were collected at interval 1 second every hours using single camera as shown in Figure 2.

\subsection{Image Analysis}

In the image analysis, the procedures consist of image rectification and timestack image analysis in which the main aim is to obtain physical information from timestack images. In the Hasaki site, the camera took successive snapshot images around Hasaki pier at the interval of 1 second. To extract pixel lines from successive snapshot images, the image coordinate of pixel $(\mathrm{u}, \mathrm{v})$ on the snapshot image need to convert on the real coordinate system $(\mathrm{x}, \mathrm{y}, \mathrm{z})$. In this rectification, the relationship between image coordinate and real coordinate as described by Holland et al, 1997 was used. The result of rectification image from snapshot image is shown in Figure 3 (middle image).

Then timestack images were collected hourly at each point in the array which can be expressed as I (xi,yi,t), where xi,yi are the spatial coordinate of the ith image pixel, and t is discrete sampling time. An example of time series of brightness intensitiy of pixel for crossshore at $\mathrm{y}=120 \mathrm{~m}$ is presented in Figure 2 (right image). 


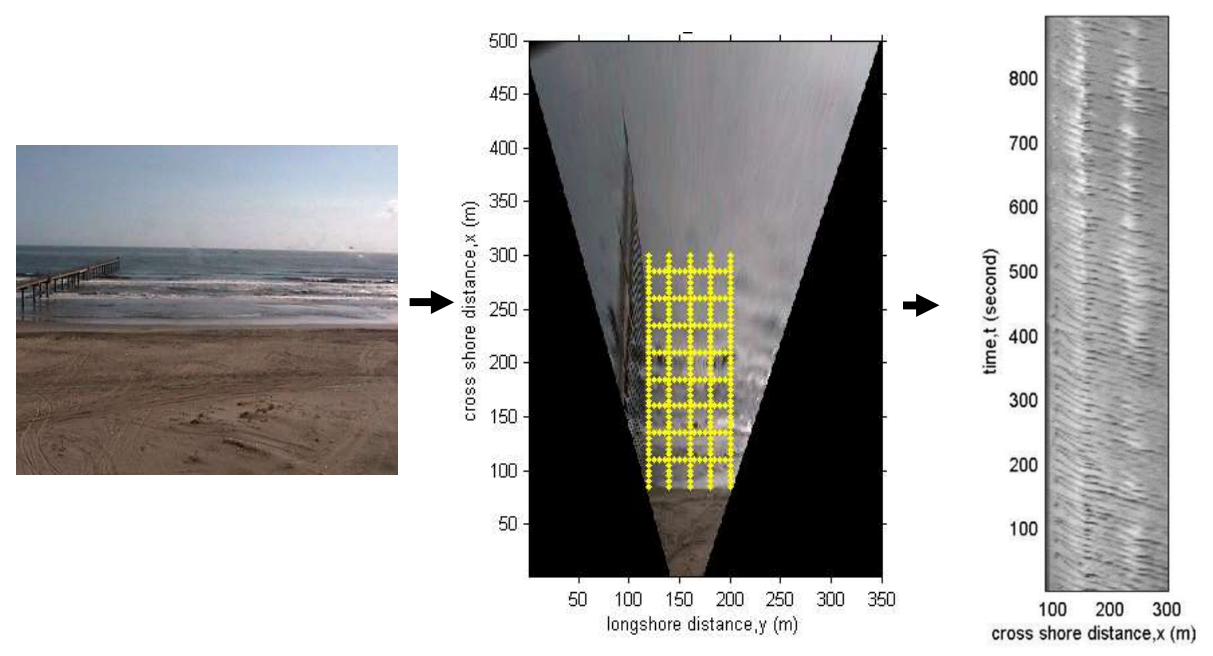

Fig. 3 Rectified image from snapshot image on 25/08/2006 at 07.00 around pier area with five crossshore arrays and eight long-shore arrays (shown with dots pixel)

\subsection{Inversion Method}

In the present research we are interested in the estimation of nearshore bathymetry from video images. The technique is based on wave number component where it can be derived from the intensity of the brightness at each pixel in the images by using cross-spectral correlation approach [8].

The mathematical formulation for cross-spectral correlation model between two pixels is described by

$C_{i, j, f}^{M O D E L}=\exp \left\{2 \pi \Delta x \sqrt{-1} \sum_{m=1}^{M} D_{i, j, m} k_{m, f} \cos \left(\alpha_{m, f}\right)\right\}$

where $f$ is wave frequency, $\Delta x$ is spacing between pixels, $D$ is design matrix defined on both sample domain $(x i, x j), \alpha$ (wave direction) and $k$ (wave number) as unknown model parameters.

The sample design matrix, $D$ is designed as basis function:

$D_{i, j, m}=\sum_{i^{\prime}=i}^{j} a_{i, m}$

where $a_{i}, m$ is smoothing weight of Hanning filter

$$
\begin{gathered}
a\left(r_{i, m}\right)=\left\{1-\cos \left(0.5 \pi\left[1+r_{i, m}\right]\right)\right\}^{2} \\
r_{i, m}=\left|x_{i}-x_{m}\right| L_{x}^{-1}
\end{gathered}
$$


where $L_{x}$ is smoothing lengths scale. Using observation data from timestack images, crossspectral observation data can be acquired by applying discrete Fourier transform to the observation to compute the cross-spectra between two pixels (sensors pair) [9].

$$
C_{i, j, f}^{O B S}=\left\langle\tilde{I}\left(x_{i}, f\right) \tilde{I} *\left(x_{j}, f\right)\right\rangle
$$

where the tildes indicate the Fourier transform, the asterisk indicates complex conjugate, angle brackets indicate ensemble or band averaging.

Since the wave number is nonlinearly related to the cross-spectral correlation as shown by equation above, a non-linear inversion method Levenberg-Marquardt [10] is used to minimize the weighted squared difference between successive estimates of the model and the observations.

$$
\Delta C_{i, j, f}^{\tau}=\left\{\gamma_{i, j, f} C_{i, j, f}^{M O D E L(\tau)}-C_{i, j, f}^{O B S}\right\}
$$

where, at each iteration $\tau$, the model-observation mismatch is weighted by the observed coherence, $\gamma_{i, j, f}$.

The minimization of cross-spectral function with Levenberg-Marquardt (LM) method consists on constructing an iterative procedure that starts with an initial value guess $k_{o}$. Then, a new estimate of wave number model is obtained with:

$$
k_{f, m}^{\tau+1}=k_{f, m}^{\tau}+\Delta k_{f, m}^{\tau}
$$

where the variation of $\Delta k_{f, m}^{\tau}$ calculated from

$$
\Delta k_{f, m}^{\tau}=\left(\left[R^{\tau}\right]^{T} R^{\tau}+\lambda^{\tau} I\right)^{-1}\left[R^{\tau}\right]^{T} \Delta C_{i, j, f}^{\tau}
$$

where $\lambda^{\tau}$ is the damping parameter, $I$ is the identity matrix and $R$ is sensitivity matrix for the cross-spectral correlation as describe:

$$
R^{\tau}=\gamma_{i, j, f} \sqrt{-1} D_{i, j, m} C_{i, j, f}^{M O D E L(\tau)} \Delta x
$$

The iteration procedure to calculate $\Delta k_{f, m}^{\tau}$ and $k_{f, m}^{\tau+1}$ from Eq. 6 and 7 is continued until the convergence criterion 
$\left|\Delta k_{f, m}^{\tau}\right|<\varepsilon$

is satisfied, where $\varepsilon$ in this model is $10^{-6}$

\subsection{Bathymetry Inversion}

The bathymetry inversion method based on timestack method computes water depth by relating wave number parameters $k$ using a suitably accurate dispersion equation. Water depth $h$ is related to local wave number $k$ and frequency $f$ through the dispersion relationship in the linear wave theory [13]

$(2 \pi f)^{2}=g k \tanh (k h)$

where $g$ is gravitational acceleration and $h$ is local water depth.

Given a value for $f$ (sample wave frequencies) and an initial depth, $h$, this equation can be solved iteratively for wave number. The Levenberg-Marquardt non-linear inverse method was used again to minimize error between the wave number predicted by Eq.10 and the wave number estimated form images derived from Eq.6.

\section{Analysis and Results}

This technique was applied using timestack data collected on August 25, 2006. Using this timestack, the wave number estimate was computed at a series of wave frequencies ranging from $0.08 \mathrm{~Hz}$ to $0.11 \mathrm{~Hz}$. It is expected to find suitable frequency from those wave frequency resolutions; the optimum wave component will give strong signal for the analysis of brightness intensity of pixel time series. On this date, the peak wave period based on field measurement was $9.1 \mathrm{sec}$; the wave direction was approach from 81 degree from North direction; and the significant wave height was $1.11 \mathrm{~m}$.

Using the measured bathymetry and the tidal level at the time of the image collection, the wave number estimate was computed for each frequency. The non-linear inversion method of Levenberg-Marquardt was applied to the sample cross-spectral correlation at each frequency over entire array. Figure 4 shows the results of the wave number estimates for each sample frequency. The best of wave number estimates were obtained at frequency $0.09 \mathrm{~Hz}$ which showed highest coherence with rms error $0.0342 \mathrm{~m}-1$ as shown in Table 1. This frequency, $0.09 \mathrm{~Hz}$ corresponds with the peak period based on field measurement.

The result of bathymetry inversion using video images data collected on August 2006 is shown in Fig 6 . The average bathymetry value estimated by inverted all frequency shows that the Hasaki beach have sand bar at $\mathrm{x}=200-200 \mathrm{~m}$. To evaluate bathymetry inversion model, the result is compare to the survey measurement data. It shows that the performance of the prediction is most accurate near shoreline and sand bar, where the differences between estimated water depth and survey water depth is less than $10-30 \mathrm{~cm}$. 

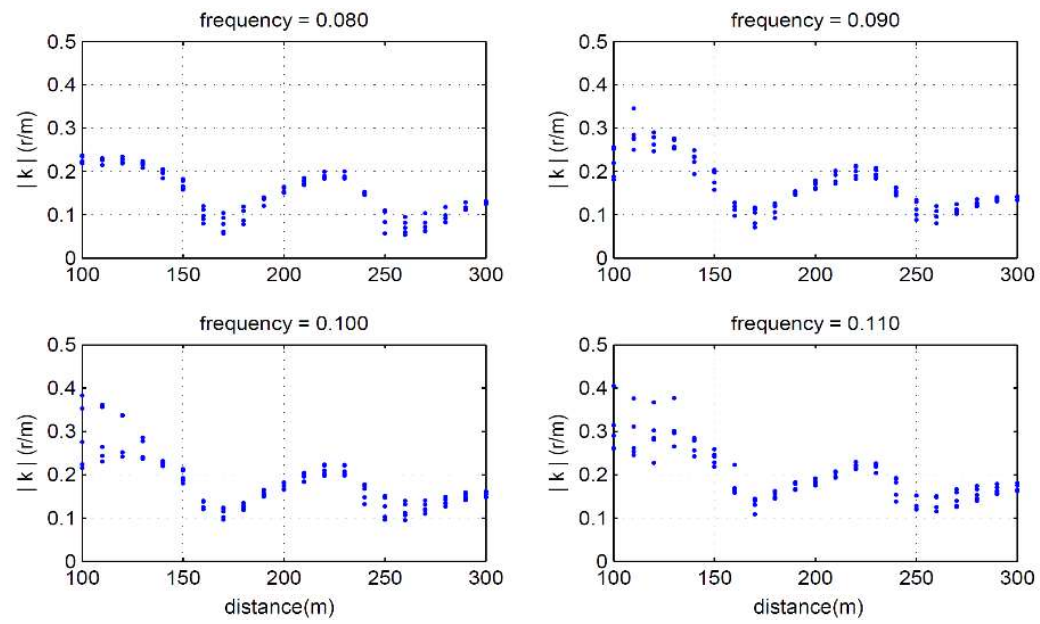

Fig. 4 Wave number estimates using cross-spectral correlation from snapshot image at 25/08/2006

Table 1. Coherence and RMS Error Wave number

\begin{tabular}{|c|c|c|c|}
\hline No & Frequency & Coherence & RMS wave number error \\
\hline 1 & 0.08 & 128.745 & 0.0358 \\
\hline 2 & 0.09 & 132.153 & 0.0342 \\
\hline 3 & 0.10 & 131.161 & 0.0354 \\
\hline 4 & 0.11 & 118.333 & 0.0421 \\
\hline
\end{tabular}
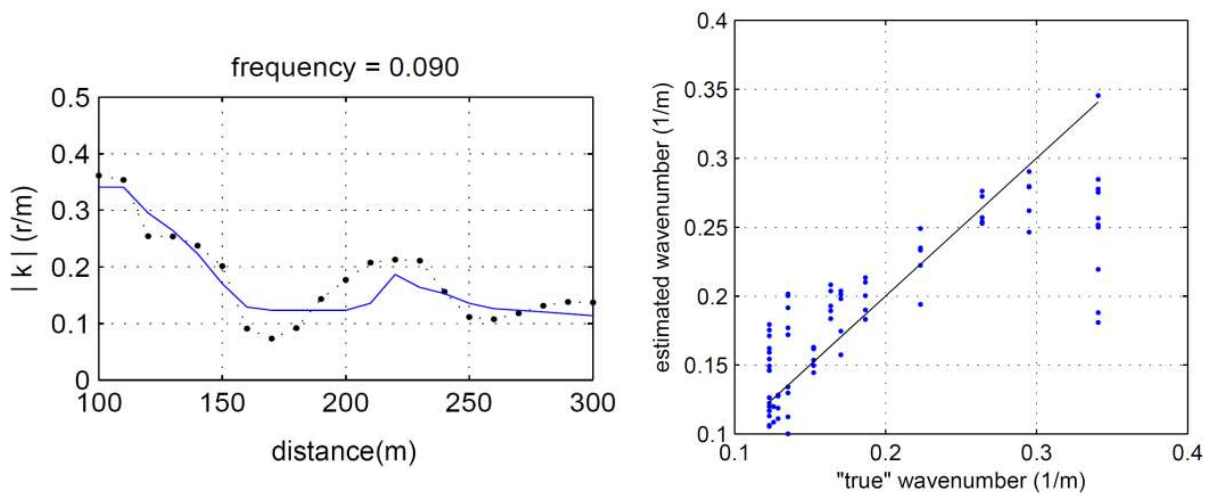

Fig. 5 (Left figure) Comparison of wave number estimated from video images (dot) and wave number from linear theory (line). Data represent from frequency 0.09 Hz. (Right figure) Correlation coefficient between wave number estimated and "true" wave number is 0.93 . 


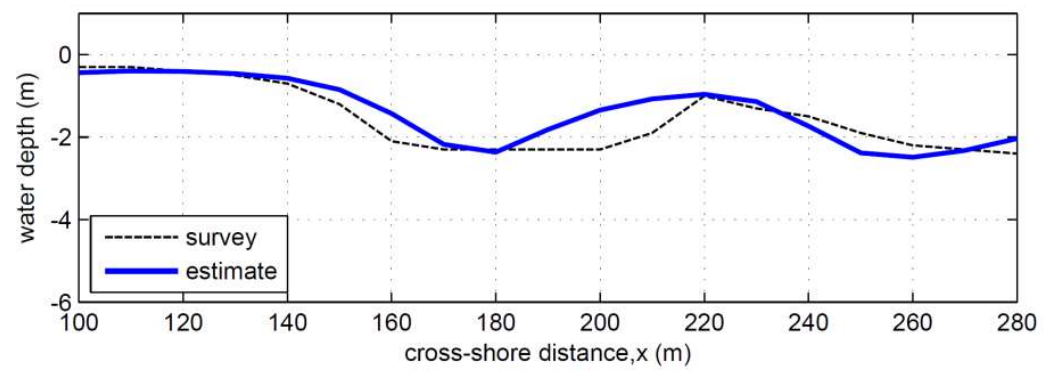

Fig. 6 Comparison between cross section profile in situ survey (dash line) and bathymetry inversion (solid line) from video images collected on August 2006.

\section{Conclusions}

An algorithm for video images sequence analysis was presented to monitor bathymetry in near shore area using non-linear inversion method to convert image pixel brightness values into depth estimates. The method consists of wave number inversion model which is based on cross-spectral correlation technique and bathymetry inversion which is based on wave dispersion model. The capability of video image technique was tested using data from Hasaki beach in Japan from August 2006 to December 2006. The results indicated that cross-spectral correlation approach have the capability to derive wave number estimate from time series of brightness intensity at each pixels to estimate bathymetry. The model showed relatively small rms errors between 0.0342-0.0421. Correlation coefficient between the estimated wave number and that from linear wave theory is 0.93 . The result of nearshore bathymetry estimates provides reasonably accurate depth estimate near shoreline and breaking area with rms 0.44 . This result indicates that nearshore bathymetry estimates can be derived from video images sequence.

\section{References}

1. Wiegel, R.L. and R.A. Fuchs. Rep. 74-9, Univ. of Calif., Berkeley (1953)

2. Greidanus, H., Hydrographic j., 83, 13-18 (1997)

3. Bell, PS., Coastal Eng, 37, 513-527 (1999)

4. Leu, L.G., Y.Y Kuo, and C.T. Lui, Coastal Eng. J, 41, 21-41 (1999)

5. Holman, R.A and J. Stanley, Coastal Engineering, 54, 477-491 (1991).

6. Holman, R.A., T.C. Lippmann, P.V. O'Neill, and K. Hathaway, Marine Geology, 97, 225-231 (1991)

7. Holland, K.T, R.A. Holman, T.C. Lipmann, J. Stanley and Plant, IEEE J. Oceanic Engineering, 22(1), pp.81-92 (1997)

8. Zikra, M., Hashimoto, N., Yamashiro, M., \& Suzuki, K. Spectral Analysis of Pixel Brightness on Video Images For Wave Analysis At Hasaki Beach, Japan. Proceedings of the Twenty-first International Offshore and Polar Engineering Conference, pp.974979 (2011).

9. Zikra, M., Hashimoto, N., Yamashiro, M., Yokota, M. and Suzuki, K. Coastal Engineering Journal, 54 (03), 24 pp., DOI: 10.1142/S0578563412500209. (2012) 
10. Bendat, J.S., and A.G. Piersol, "Random Data: Analysis \& Measurement Techniques", 566 pp., Wiley Intersci., New York (2000).

11. Press, W.H, Teukolsky, S.A, Vetterling, W.T, and Flannery, B.P., "Numerical Recipes in C: The Art of Scientific Computing”, 2nd ed, Cambridge University Press (1992).

12. Stockdon, H.F. and Holman, R.A., Journal of Geophysical Research 105, pp. 22015 22033 (2000).

13. Dean, R.G., and R. A. Dalrymple, “Water Waves Mechanics for Engineer and Scientist”, 353 pp., World Sci., River Edge, N.J (1991). 